\title{
Influence of spatial variations of the geoelectric field on geomagnetically induced currents
}

\author{
Ari Viljanen ${ }^{1, *}$ and Risto Pirjola ${ }^{1,2}$ \\ ${ }^{1}$ Finnish Meteorological Institute, Erik Palménin aukio 1, 00560 Helsinki, Finland \\ ${ }^{2}$ Geomagnetic Laboratory, Natural Resources Canada, 2617 Anderson Road, Ottawa, Ontario K1A 0E7, Canada
}

Received 31 May 2017 / Accepted 30 August 2017

\begin{abstract}
The geoelectric field driving geomagnetically induced currents (GIC) has complex spatial variations. It follows that different patterns of the field vectors in a given area having the same regional mean can produce very different GIC. In this study, we consider a few power grid models and calculate GIC due to a modelled geoelectric field with a regional mean magnitude of $1 \mathrm{~V} / \mathrm{km}$. Altogether 8035 snapshots of the electric field are included. We also assume two different ground conductivity models, of which the simpler one consists of two layers across the whole region, and another model has four different two-layer blocks. As a measure of GIC, we use the sum of currents at all substations of the power grid. We also consider the distribution of GIC at a single site. For a given grid, differences between the two ground conductivity models are small. When comparing different power grid models, the sum of GIC varies relatively more for a grid with a small number of substations and transmission lines. When the area and the number of substations increase, the relative difference between the smallest and largest GIC sum decreases. In all cases, assuming a spatially uniform electric field leads to a reasonable estimation of the GIC magnitudes, but it does not produce the largest GIC sum. For a single substation, there is a large variety of GIC values due to different electric field configurations.
\end{abstract}

Keyword: geomagnetically induced currents

\section{Introduction}

Especially during recent years, several studies of geomagnetically induced currents (GIC) in power grids have focussed on extreme case scenarios, since large geomagnetic storms are a potential threat to power transmission (Boteler et al., 1998; Bolduc, 2002; Molinski, 2002). A common approach is to use long geomagnetic data sets to extrapolate indices (Siscoe, 1976; Love et al., 2015; Riley, 2012), or to extrapolate the amplitude of the variation field or its time derivative (Danskin \& Lotz, 2015; Love et al., 2016; Thomson et al., 2011). A related possibility is to extrapolate the geoelectric field modelled from magnetic field data (Myllys et al., 2014; Nikitina et al., 2016; Pulkkinen et al., 2012; 2015; Wintoft et al., 2016). In a few cases, there are long enough time series of the recorded electric field allowing for deriving statistics (Langlois et al., 1996). These approaches lead to local estimates at observation sites and they are not able to provide a regional or global instantaneous snapshot of electric field vectors required for full GIC modelling.

\footnotetext{
*Corresponding author: ari.viljanen@fmi.fi
}

Another way is to apply simulations of the solar windmagnetosphere-ionosphere interaction (Ngwira et al., 2013; 2014). In principle, this approach is ideal, since it produces time series of ground magnetic and electric fields. They can then be directly applied to standard GIC modelling at any geographical scales (e.g. Lehtinen \& Pirjola, 1985; Viljanen et al., 2012). However, simulations are still far from the capability to model precisely small-scale features and local enhancements that are important for GIC (cf. Pulkkinen et al., 2006; 2015). By small scales we refer to variations of the geoelectric field in the scale of $10-100 \mathrm{~km}$, which corresponds to typical lengths of power transmission lines in the region studied here. The same accuracy should be achieved for simulated ionospheric currents that primarily cause fast geomagnetic variations. Ionospheric currents are in turn very much controlled by magnetospheric processes. Since the input to simulations at the magnetospheric boundary is in practice solar wind data measured at the Lagrangian 1 point, we cannot expect to obtain high accuracy on the ground. Additionally, it is not only important to reach the correct spatial behaviour of the ground fields, but the temporal evolution is equally significant.

The geoelectric field varies rapidly temporarily and spatially as demonstrated, for example, by Beggan et al. (2013), Bedrosian and Love (2015), Wei et al. (2013) and 


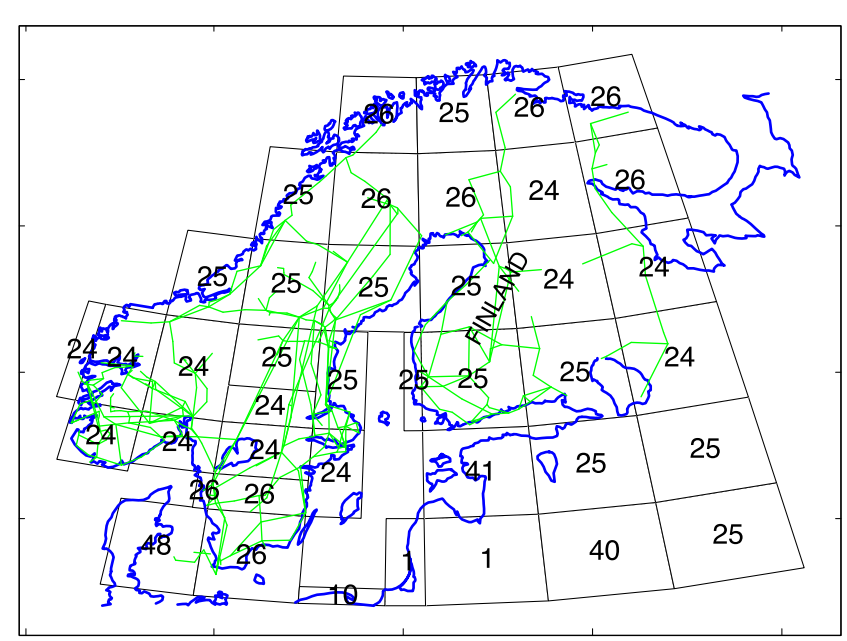

Fig. 1. Blocks of the North European conductivity map (extracted from Viljanen et al. (2014)) and the power transmission lines in the Nordic grid model (green lines). Blocks 24-26 and 48 cover the power grids considered in this paper (Table 1).

Wang et al. (2016) for regional and continental scales, and by Püthe and Kuvshinov (2013) and Ngwira et al. (2015) for global scales. Temporal variations are due to time variations of space currents and are connected with time variations of the geomagnetic field by Faraday's law. Spatial variations are caused by a nonuniform geomagnetic field and by a laterally varying ground conductivity. The former is generally the major factor affecting GIC magnitudes (Beggan, 2015). The latter leads to spatial variations of the electric field even if the magnetic field is a uniform plane wave (Bedrosian \& Love, 2015). In case of nonuniform magnetic fields, lateral variations of the ground conductivity can locally play a further significant role (Beggan, 2015).

The electric field at a single point is a useful quantity to estimate the sum of the absolute values of GIC at all substations in the surrounding power grid (Viljanen et al., 2015). However, engineering analysis of GIC impacts on single transformers requires complete modelling using as input the electric field given as horizontal vectors across the grid.

It is intuitively clear that even if the electric field at two separate time steps has an identical regional mean magnitude, the vector fields can be totally different leading to substantially different magnitudes of GIC at each substation. In this paper, we will quantify this by using an extensive geomagnetic data set and a few realistic models of high-voltage power grids. We will answer the following question: "How much does GIC vary in a power grid if the regional mean of the electric field has a fixed value, but the vector patterns are different across the grid?"

\section{Data and methods}

We will apply a straightforward standard method for modelling GIC using geomagnetic data, ground conductivity models and power grid models. For power grids, we use the following three cases, adopted from Viljanen et al. (2012):

- Finnish $400 \mathrm{kV}$ grid in 1978-1979 with 17 substations

(further on called Finland 1978-1979);
- simplified model of the present Finnish high-voltage grid (220 and $400 \mathrm{kV}$ ) with 55 substations (further on called Finland 2016);

- simplified model of the Nordic high-voltage grid (220 and $400 \mathrm{kV}$ ), also containing North-West Russia with 339 substations (further on called Nordic, see Fig. 1).

Although the two latter grid models are only approximate (Viljanen et al., 2012), they still serve for the purpose of this paper. They represent a realistic topology and have reasonable resistances. This also gives an idea how much the results depend on a specific grid model.

The electric field is calculated using a sub sample of the IMAGE network (Tanskanen, 2009) magnetometer data in 1994-2015 as 1-min values. As the ground conductivity model, we use two cases. One is the EURISGIC model (Adam et al., 2012; Viljanen et al., 2014) in which there are four different 1-D ground structures next to each other in the region considered here (Fig. 1, Table 1). Another case applies a single 1-D model for the whole region, and here we have selected the model of block 25 . Concerning the conductivity blocks in more detail, all of them have the same quite high resistivity in the upper layer. This generally indicates the possibility for large electric fields. Since block 24 has the thickest upper layer, it evidently has the largest electric field under the assumption of equal magnetic field variations. Block 25 has the smallest resistivity in the lower layer, which results in smaller electric fields.

Following the method by Viljanen et al. (2012), we calculate the horizontal electric field vectors (E) at the nodes (substations) of the power grids. For each day, we save the values at the time step when the sum of GIC at all nodes reaches its maximum. Taking only one value per day obviously gives an independent set of snapshots. Then we scale $\mathbf{E}$ at these time steps to make its mean magnitude equal to $1 \mathrm{~V} / \mathrm{km}$. This makes different time steps directly comparable. Altogether, there are 8035 snapshots of $\mathbf{E}$ vectors. We note that the electric field is determined only at the nodes of the power grid. To calculate the voltage along each transmission line, we need to interpolate the electric field as described in Viljanen et al. (2012).

We do not try to assess whether all snapshot patterns could also occur in reality, with the assumption that the mean field should reach the relatively large value of $1 \mathrm{~V} / \mathrm{km}$. For this study it is more relevant to simulate a large number of different vector patterns with the same regional mean. The largest modelled unscaled electric field at a single substation in the Nordic countries is $1.7 \mathrm{~V} / \mathrm{km}$ in $1994-2015$ when using the full ground conductivity model. For comparison, Langlois et al. (1996) reports the maximum measured value of $1.7 \mathrm{~V} / \mathrm{km}$ in Québec, Canada, within a 500-day period, in an area that corresponds to South Finland in geomagnetic latitudes. Model calculations in North America by Wei et al. (2013) and Nikitina et al. (2016) indicate values up to $2-5 \mathrm{~V} / \mathrm{km}$. We remind that the electric field is very sensitive to the conductivity, so different ground models can produce fields with clearly different magnitudes when the same magnetic field is used as input.

\section{Results}

The diurnal distribution of the electric field snapshots as defined in Section 2 is shown in Figure 2 for the Finland 2016 


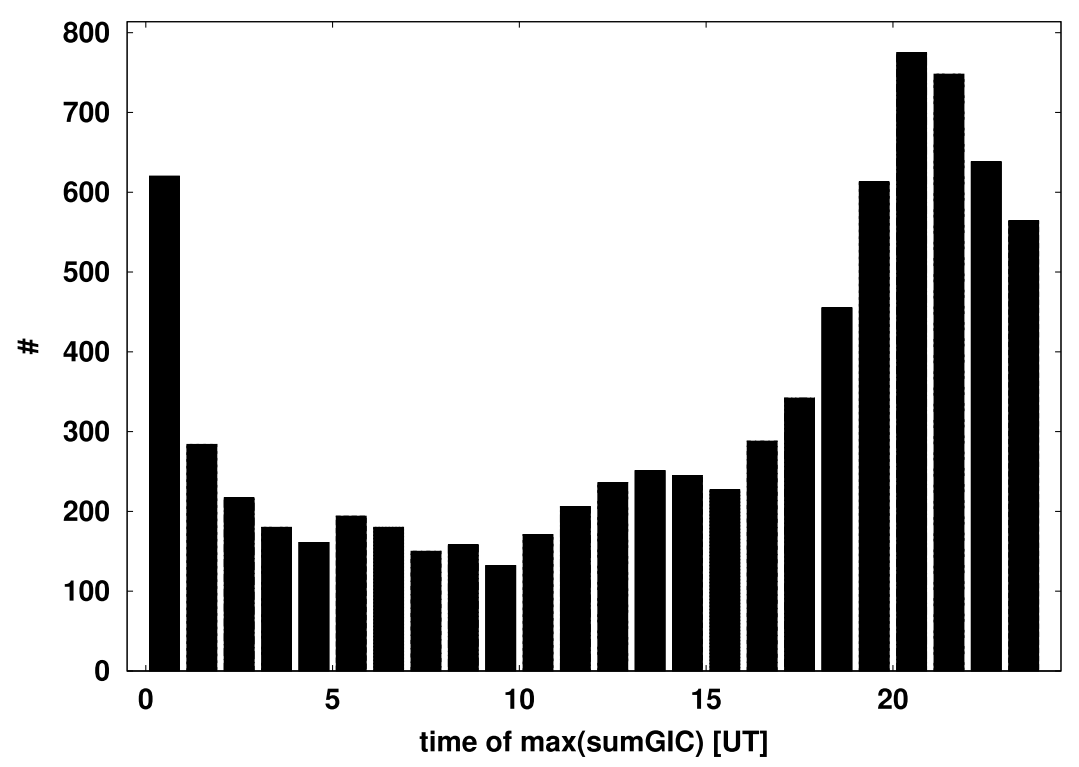

Fig. 2. Daily distribution of the electric field vector snapshots used in this study. Grid model: Finland 2016, full ground conductivity model (Table 1).

Table 1. Layered models of the ground conductivity applied in the Nordic countries. Layer thickness and resistivity (=inverse of conductivity) are $d$ and $\rho$, respectively. Block numbering refers to Figure 1. Source of the model parameters: http://real.mtak.hu/2957 (available also as supplementary material in Viljanen et al. (2014)). Block 48 is relevant only when the Nordic grid model is considered.

\begin{tabular}{llllllll}
\hline & \multicolumn{2}{c}{ Block 24 } & \multicolumn{3}{c}{ Block 25 } & & \multicolumn{2}{c}{ Block 48 } \\
\hline$d(\mathrm{~km})$ & $\rho(\Omega \mathrm{m})$ & $d(\mathrm{~km})$ & $\rho(\Omega \mathrm{m})$ & $d(\mathrm{~km})$ & $\rho(\Omega \mathrm{m})$ & $d(\mathrm{~km})$ & $\rho(\Omega \mathrm{m})$ \\
\hline 200 & 5000 & 30 & 5000 & 30 & 5000 & 5 & 10 \\
Inf & 200 & Inf & 20 & Inf & 200 & 30 & 190 \\
& & & & & 100 & Inf \\
\hline
\end{tabular}

grid model. For other grid models, it is very similar. There is a maximum close to the local midnight (about $22 \mathrm{UT}$ ), which is expected (cf. Viljanen, 2001). The effect of the ground conductivity model on the diurnal distribution is very small, $\mathrm{i}$. e. the single-block model gives a nearly identical result.

Figures 3 and 4 show snapshots of the modelled electric fields and GIC in Finland. In Figure 3, a single ground conductivity model (block 25 ) is applied across the whole area, so all nonuniform features of the electric field are associated with spatial variations of the magnetic field. In Figure 4 with the full conductivity model, the high-resistive area in the north (block 24) leads to an enhanced electric field there. Pulkkinen et al. (2015) present sample plots of the modelled electric field close to and within the same region, demonstrating notable spatial variations when a single conductivity model was assumed (different from the one applied here).

Just by looking at the electric field patterns, it is not immediately clear how large GIC sums could be expected. In Figure 3 in the two rightmost plots corresponding to the smallest GIC sums, it may seem puzzling that GIC remains small despite the large electric fields in the north. However, field enhancements are concentrated on a limited area and elsewhere the field is small. In the two leftmost plots showing the largest GIC sums, the field is larger in the area where most substations are located.
For reference, Table 2 gives the GIC sums in case of a uniform electric field of $1 \mathrm{~V} / \mathrm{km}$ across the whole power grid. The basic cases are a uniform field to the north or to the east, since from them GIC due to a uniform field to any direction can be calculated as a linear superposition. Especially, we can easily find the directions of the field that produce the smallest and largest sum of GIC (Arajärvi et al., 2011). For this simple structure of the electric field, there is a fairly small dependence of the GIC sum on the direction of the field: the maximum GIC in Table 2 is $5-20 \%$ larger than the minimum. This difference decreases when the size of the grid increases. The directions given in Table 2 vary much depending on the power grid. Consequently, there is no clear directional preference compared, for example, to the geomagnetic north that is tilted about $10 \circ$ westward from the geographic north in this region.

Table 3 lists the largest and smallest sums of GIC modelled for the three different power grids and the two different ground conductivity models. We first note that for a given power grid, the range of values is nearly the same independently of the ground conductivity model. This is evidently due to the constraint set to the electric field to always have the regional mean of $1 \mathrm{~V} / \mathrm{km}$. Concerning the ratio between the largest and smallest GIC sum, it is largest (about 2.4) for the Finland 1978-1979 grid with only 17 substations, and decreasing to 

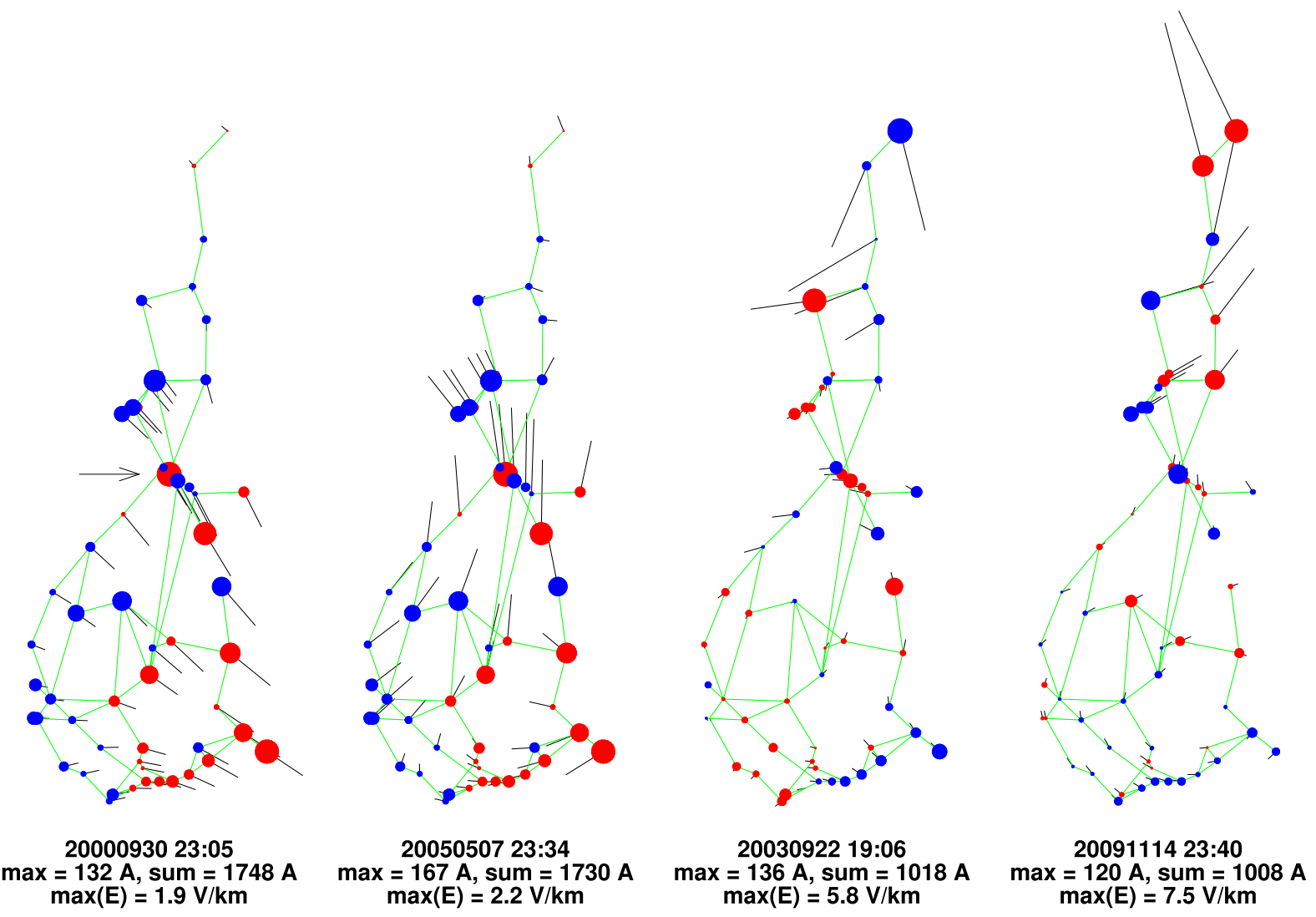

Fig. 3. Snapshots of the modelled electric field (arrows) and GIC (dots) at the nodes of the Finland power grid. Ground conductivity model of block 25 is used for the whole area (Table 1). The mean value of the electric field is set to $1 \mathrm{~V} / \mathrm{km}$. GIC from the grid into the ground is plotted by red and from the ground into the grid by blue. Two leftmost plots: cases of the two largest GIC sums (Table 3). Two rightmost plots: cases of the two smallest GIC sums. Note that the scale of the electric field is different in each plot. The black arrow in the leftmost plot shows the location of the Pikkarala substation considered separately in Figure 7. Transmission lines are plotted by green.

about 1.5 for the Nordic grid with 339 substations. This is understandable when first considering the simplest grid consisting of only one straight line grounded at both ends and assuming a uniform electric field. Then GIC varies from zero (field perpendicular to the line, causing a zero voltage between the end points) to a maximum value (field parallel to the line). An increasing number of substations and lines creates a more complex geometry. Then the minimum GIC obviously clearly differs from zero, since there is hardly an electric field that happens to be perpendicular to all transmission lines.

Comparison of Tables 2 and 3 shows that a spatially uniform electric field $(1 \mathrm{~V} / \mathrm{km})$ gives a reasonable indicator of the magnitude of GIC sums. However, a field varying spatially, with a regional mean of $1 \mathrm{~V} / \mathrm{km}$, can produce GIC sums that are about $15-56 \%$ larger than due to a spatially uniform field. On the other hand, the nonuniform field can also produce GIC sums which are $12-34 \%$ smaller than the smallest value due to a uniform field. These differences are largest for the simple grid of Finland 1978-1979, but even for the Nordic grid, the maximum due to a nonuniform field is $31 \%$ larger than due to a uniform field.

The histograms of GIC sums in different power grids and for different ground models are shown in Figure 5. As already noted, the distribution is relatively widest for the small Finnish grid in 1978-1979. There are some small differences in the shape of the distributions between the conductivity models.
The most prominent difference occurs with the Nordic grid for which the full ground model gives larger GIC sums (mean 7273 A versus 6790 A by the single-block model).

For an impression of the occurrence of GIC in a power grid, we checked which nodes experience the largest current at the 8035 time steps studied here. Table 4 lists the top nodes in the Finland 2016 grid (containing altogether 55 nodes) in case of both ground conductivity models of Table 1. For comparison, we also show the largest GIC that would be produced by a uniform electric field of $1 \mathrm{~V} / \mathrm{km}$ across the whole grid. Figure 6 illustrates the occurrence on the map. For both ground conductivity models, there is a small region in the north where the largest GIC tend to occur. These nodes are favourable to have large GIC, as indicated by the values based on a uniform electric field. Additionally, the electric field is usually larger at higher latitudes. In case of the full ground conductivity model, this is even more prominent. Then the smaller conductivities in the north produce further enhancement of the electric field compared to the case of the single 1-D layered model applied everywhere. These findings are basically the same as mentioned by Myllys et al. (2014) concerning a model study of the Norwegian power grid.

As a more detailed example of a single substation, we consider Pikkarala (64.9 N, 25.8 E, see Fig. 3) assuming the Finland 2016 grid model. Large GIC values are likely there, since a uniform northward electric field of $1 \mathrm{~V} / \mathrm{km}$ would 

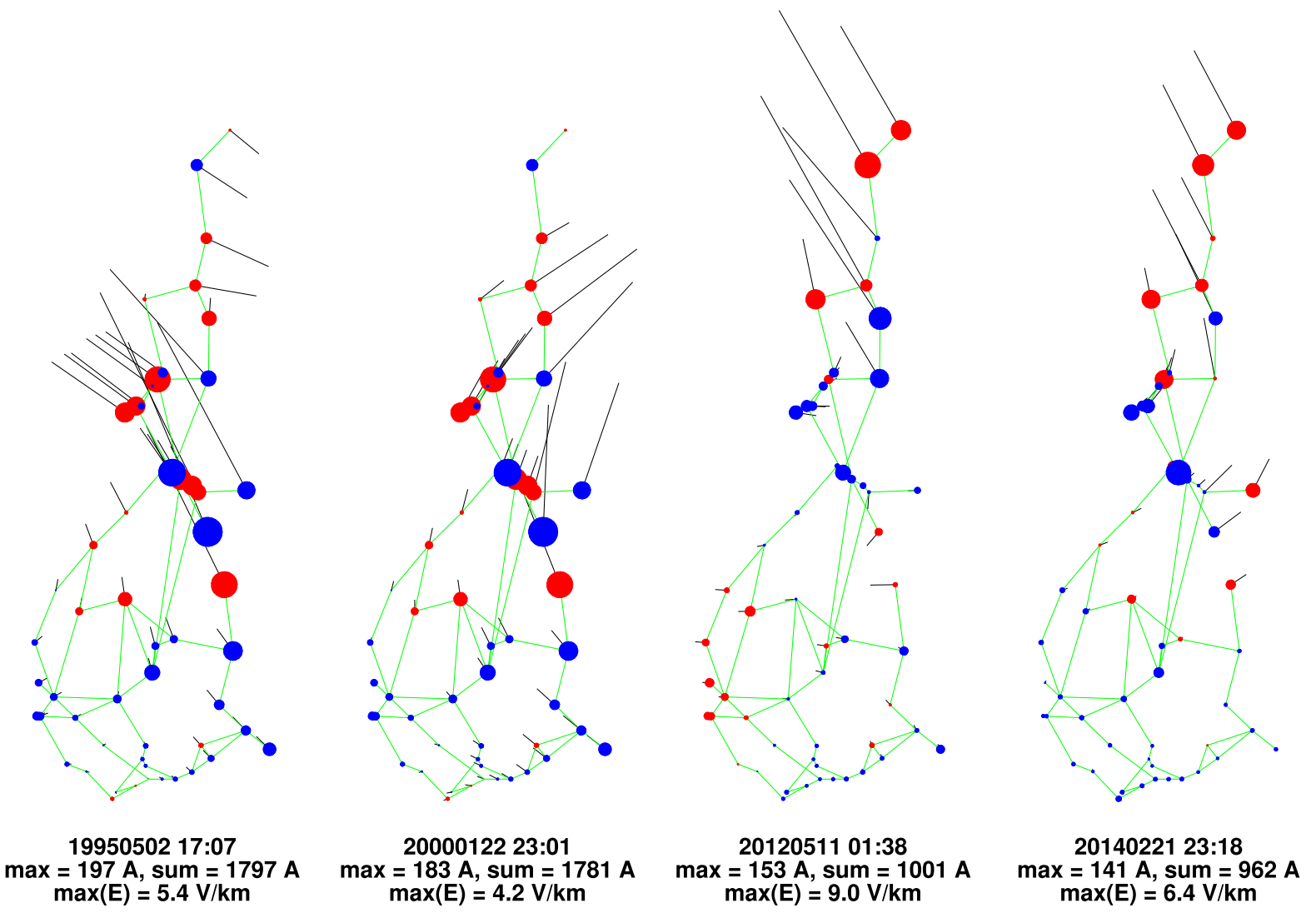

Fig. 4. As Figure 3 for the cases of the two largest and smallest GIC sums in Table 3, when the full ground conductivity model is used.

Table 2. Sum of the absolute values of GIC (A) due to a uniform electric field (1 V/km) at the substations in the model grids. Four cases of the field are considered: northward, eastward, the field in the direction $\left(\alpha_{\min }, \alpha_{\max }\right)$ producing the smallest and largest sums of GIC. The direction is measured in degrees clockwise from the north.

\begin{tabular}{lllllll}
\hline & North & East & Min & $\alpha_{\min }$ & $\alpha_{\text {max }}$ \\
\hline Finland 1978-1979 & 657 & 722 & 624 & 16 & 118 \\
Finland 2016 & 1465 & 1424 & 1401 & 76 & 147 \\
Nordic & 6868 & 6653 & 6638 & 83 & 1568 \\
\hline
\end{tabular}

produce GIC of 107.3 A, and an eastward field would cause 11.7 A. It follows that the maximum GIC due to a uniform electric field of $1 \mathrm{~V} / \mathrm{km}$ at an optimal direction would be $\sqrt{107.3^{2}+11.7^{2}} \mathrm{~A}=107.9$ A (cf. Arajärvi et al., 2011). As Figure 7 shows, the distribution is clearly broader than for the GIC sum of the whole grid, when the standard deviation is percentually compared to the mean. A wide range of values from about zero up to larger than $200 \mathrm{~A}$ can occur at Pikkarala. So in this case, GIC estimated by a uniform electric field (1 V/ $\mathrm{km}$ ) is less than half of the largest value that a nonuniform field could cause under the assumption of the mean equal to $1 \mathrm{~V} / \mathrm{km}$. As Table 4 shows, the maximum GIC due to a nonuniform field can be relatively even much larger. This is obviously due to a pronounced local enhancement of the electric field, and this underlines the need to understand localised features in addition to regional scales (cf. Ngwira et al., 2015; Pulkkinen et al., 2015).
Finally, we made an experiment by restricting the selection of data to the hour of 20-21 UT. This is a little before the local midnight, when substorms regularly occur. We used the Finland 2016 grid model and the ground model of block 25 everywhere. The range of the GIC sum varies from 1015 to $1784 \mathrm{~A}$, which is nearly equal to the range in Table 3 . The four top nodes in Table 4 also occur in the same order. The mean and standard deviation of GIC at Pikkarala become $69 \pm 40 \mathrm{~A}$, i.e. nearly equal to Figure $7(72 \pm 44 \mathrm{~A})$.

\section{Discussion}

Nonuniform geoelectric fields resulting from complex ionospheric-magnetospheric currents have already been investigated based on model calculations a long time ago (e.g. Price, 1962; Hermance \& Peltier, 1970; Weaver, 1973; 
Table 3. The five largest and the five smallest sums of GIC due to an electric field scaled to the mean of $1 \mathrm{~V} / \mathrm{km}$ in the area of three different power grids. One-minute geomagnetic data of 1994-2015 are used. Two different ground conductivity models given by Table 1 are applied.

\begin{tabular}{|c|c|}
\hline & $\operatorname{Sum}(\mathrm{GIC})(\mathrm{A})$ \\
\hline \multicolumn{2}{|c|}{ Finland 1978-1979 ground model of block 25 day and time (UT) } \\
\hline $2015041000: 53$ & 1164.1 \\
\hline $2012042823: 19$ & 1156.8 \\
\hline $2010012016: 50$ & 1155.8 \\
\hline $2013052519: 56$ & 1155.7 \\
\hline $2014030122: 10$ & 1141.6 \\
\hline \multicolumn{2}{|l|}{$\ldots$} \\
\hline $2009010100: 00$ & 538.7 \\
\hline $2001102218: 17$ & 525.0 \\
\hline $2012111021: 35$ & 522.7 \\
\hline $1996100100: 00$ & 483.2 \\
\hline $1996093023: 59$ & 481.2 \\
\hline \multicolumn{2}{|c|}{ Finland 1978-1979 3-block ground model day and time (UT) } \\
\hline $2004060321: 36$ & 1141.5 \\
\hline $2013052519: 56$ & 1136.0 \\
\hline $2010041201: 18$ & 1109.3 \\
\hline $2015060100: 31$ & 1076.1 \\
\hline $2011061023: 15$ & 1075.5 \\
\hline \multicolumn{2}{|c|}{$\ldots$} \\
\hline $2012061622: 50$ & 536.5 \\
\hline $1996100100: 00$ & 499.7 \\
\hline $1999092222: 23$ & 489.0 \\
\hline $1996093023: 59$ & 486.5 \\
\hline $1995021023: 12$ & 476.2 \\
\hline \multicolumn{2}{|c|}{ Finland 2016 ground model of block 25 day and time (UT) } \\
\hline $2000093023: 05$ & 1747.6 \\
\hline $2005050723: 34$ & 1730.1 \\
\hline $1998021722: 58$ & 1701.9 \\
\hline $2015122016: 18$ & 1698.8 \\
\hline $1995050217: 07$ & 1695.5 \\
\hline \multicolumn{2}{|l|}{$\ldots$} \\
\hline $2011092902: 28$ & 1055.2 \\
\hline $2014021122: 06$ & 1039.7 \\
\hline $1995041323: 43$ & 1038.6 \\
\hline $2003092219: 06$ & 1017.8 \\
\hline $2009111423: 40$ & 1007.7 \\
\hline \multicolumn{2}{|c|}{ Finland 2016 3-block ground model day and time (UT) } \\
\hline $1995050217: 07$ & 1797.3 \\
\hline 20000122 23:01 & 1780.6 \\
\hline $2004110723: 31$ & 1771.3 \\
\hline $2003072700: 40$ & 1701.0 \\
\hline 20000406 18:09 & 1690.9 \\
\hline \multicolumn{2}{|l|}{$\ldots$} \\
\hline $2007041500: 08$ & 1035.4 \\
\hline $2012053000: 14$ & 1019.9 \\
\hline $2014090321: 11$ & 1007.1 \\
\hline $2012051101: 38$ & 1000.9 \\
\hline $2014022123: 18$ & 962.0 \\
\hline \multicolumn{2}{|c|}{ Nordic ground model of block 25 day and time (UT) } \\
\hline $2013071500: 30$ & 8618.4 \\
\hline $2012071201: 09$ & 8485.0 \\
\hline $2006032117: 42$ & 8454.2 \\
\hline
\end{tabular}

Table 3. (continued).

\begin{tabular}{|c|c|}
\hline & $\operatorname{Sum}(\mathrm{GIC})(\mathrm{A})$ \\
\hline $2009062902: 02$ & 8375.4 \\
\hline $2015081600: 02$ & 8364.2 \\
\hline \multicolumn{2}{|l|}{$\ldots$} \\
\hline $2001082221: 36$ & 5949.5 \\
\hline $2003052922: 27$ & 5909.1 \\
\hline $2008032818: 05$ & 5882.7 \\
\hline $1999111721: 13$ & 5872.5 \\
\hline $2003033020: 15$ & 5871.5 \\
\hline \multicolumn{2}{|c|}{ Nordic 3-block ground model day and time (UT) } \\
\hline $2009062902: 03$ & 9088.4 \\
\hline $20060321 \quad 17: 42$ & 9087.3 \\
\hline $2015100912: 10$ & 8785.3 \\
\hline $2015081600: 02$ & 8744.5 \\
\hline $2004111217: 54$ & 8730.3 \\
\hline \multicolumn{2}{|l|}{$\ldots$} \\
\hline $2014022123: 18$ & 6233.6 \\
\hline $1998111402: 22$ & 6233.4 \\
\hline $1998041122: 10$ & 6232.0 \\
\hline $1998050503: 57$ & 6231.0 \\
\hline $2009121320: 20$ & 6191.2 \\
\hline
\end{tabular}

Hibbs \& Jones, 1978; Häkkinen et al., 1989; Pirjola \& Häkkinen, 1991; Viljanen et al., 1993; 1999). The principal aim of these studies has, however, been to understand the effects of nonuniform sources on magnetotelluric studies of the Earth's conductivity structure. Pirjola et al. (2000) examined nonuniform geoelectric fields regarding especially applications to GIC calculations. Zheng et al. (2013) investigated geoelectric field calculations based on an ionospheric line current source or on a plane wave model. They showed that, in some cases, these two models may lead to significantly different geoelectric field values, which can cause inaccuracies in connection with GIC studies.

Pirjola and Viljanen $(1989 ; 1991)$ considered GIC in the Finnish $400 \mathrm{kV}$ power grid due to an east-west ionospheric line current, which causes an eastward electric field. The Earth structure was assumed to be very simple consisting of a nonconducting surface layer above a perfect conductor. They scaled the amplitude of the line current so that the mean electric field was $1 \mathrm{~V} / \mathrm{km}$ at the power grid. This was compared to the case of a uniform eastward electric field of $1 \mathrm{~V} / \mathrm{km}$. From Table I of Pirjola and Viljanen (1989), the sum of GIC at all nodes is $676.0 \mathrm{~A}$ for the uniform field and 991.8 $\mathrm{A}$ for the line current field. So this simple example of Pirjola and Viljanen (1989) also demonstrates that the uniform field does not produce the largest possible GIC.

An additional detail in the study made by Pirjola and Viljanen (1991) was to understand how much GIC extend all over a power grid if a nonuniform geoelectric field only affects a part of the grid. Pirjola (2010) investigated this even more systematically. The conclusion from those studies was that GIC do not flow long distances in a power network. This also means that a very localised enhancement of the electric field does not cause large GIC in a large area, as Figure 3 also demonstrates. 

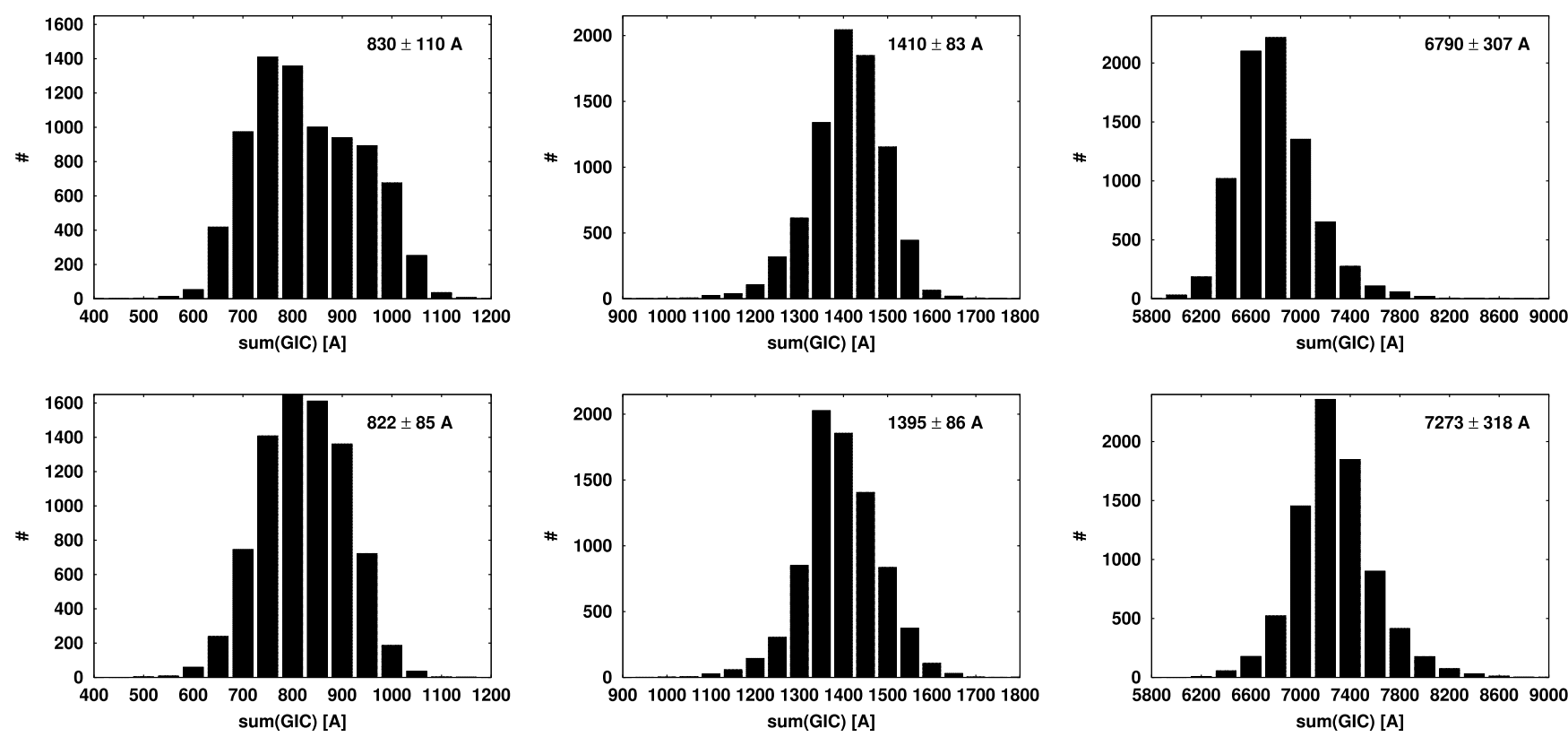

Fig. 5. Top: distribution of the sum of GIC at all nodes of the power grid using the ground conductivity model of block 25 (Table 1). Bottom: as the top row, but using the full ground model of Table 1. Grid models from the left: Finland 1978-1979, Finland 2016, Nordic. The mean and standard deviation are shown in the upper right corner of each plot.

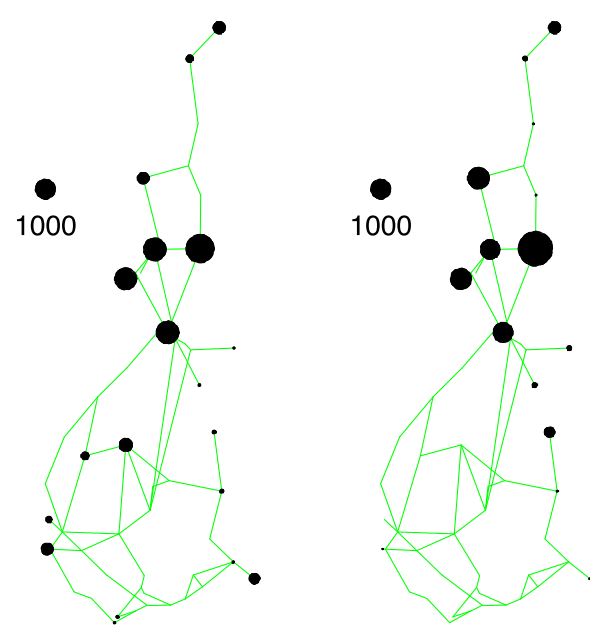

Fig. 6. Number of the time steps at which the largest GIC occurs at a given node. Grid model: Finland 2016, ground conductivity model: block 25 (left), full model (right). The separate black dot shows the scale and the number of time steps is proportional to the area of the dot. See also Table 4.

\section{Conclusions}

We have calculated GIC in three power grids driven by a modelled geoelectric field that has a fixed mean magnitude of 1 $\mathrm{V} / \mathrm{km}$, but different vector patterns across the grid. Altogether 8035 snapshots of the electric field were used. Additionally, we have assumed two different ground conductivity models, of which the simpler one consists of two layers across the whole region. Another ground model has four different two-layer blocks. As a measure of GIC, we use the sum of induced currents at all substations of the power grid. We also consider where the maximum GIC occurs at each snapshot, and discuss the distribution of GIC values at a single substation. As the basic reference value, we use GIC produced by a uniform electric field of $1 \mathrm{~V} / \mathrm{km}$.

The main finding is that, compared to the case of a spatially uniform electric field of $1 \mathrm{~V} / \mathrm{km}$, the sum of GIC has a clearly broader range when the driving field is nonuniform, but constrained to a regional mean value of $1 \mathrm{~V} / \mathrm{km}$. There is only a weak dependence on the ground conductivity model, so forcing the regional mean to a fixed value is obviously quite a dominating factor. Of the highest interest are the largest GIC sums, which exceed the maximum due to a uniform field by $15-56 \%$. These differences are largest for a grid having only 17 nodes, but even for a grid of 336 nodes, the difference is $31 \%$.

When single substations are considered, there are only a few sites that tend to experience the largest instantaneous GIC. There are three contributing factors to be taken into account. First, such sites need to have a topologically favourable location, i.e. a uniform electric field must cause quite high GIC. Second, location close to geomagnetically active regions is necessary to generate a large electric field, in our case this means northern parts of a power grid. Third, if the ground conductivity is small at these sites it further increases the electric field and GIC.

At a single site, GIC varies from about zero to some maximum, which can be substantially larger than produced by a uniform electric field. This is obviously caused by a pronounced local enhancement of the electric field. When compared percentually to the mean GIC, the standard deviation of GIC becomes much larger than for the GIC sum including all substations.

From the mathematical viewpoint, a uniform electric field is always a gradient of a scalar function, whereas a general nonuniform field is also contributed by the time derivative of a vector potential. Physically, this leads a rich variety of patterns 

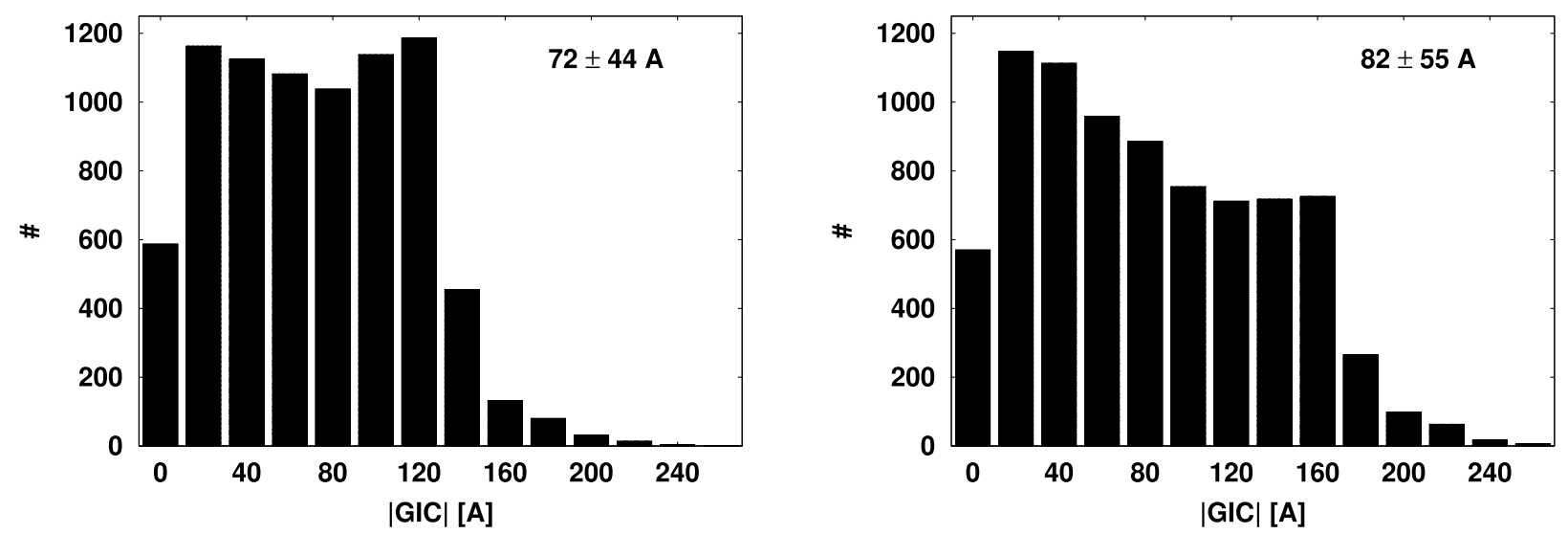

Fig. 7. Distribution of the absolute value of GIC at one node of the Finland 2016 power grid (Pikkarala; 64.9 N, 25.8 E) using the ground conductivity model of block 25 (left) and the full ground model (right) (Table 1). The mean and standard deviation are shown in the upper right corner of each plot.

Table 4. Top 10 nodes in the Finland 2016 grid at which the largest GIC occurs during the 8035 time steps used in this study. The largest GIC due to a uniform electric field of $1 \mathrm{~V} / \mathrm{km}$ across the whole grid is given in the last column $\left(\max \left(\mathrm{GIC}_{0}\right)\right)$. The two different ground conductivity models of Table 1 are used. See Figure 6 for graphical illustration.

\begin{tabular}{|c|c|c|c|c|}
\hline & (lat,lon) & Hits & $\operatorname{Max}(\mathrm{GIC})(\mathrm{A})$ & $\operatorname{Max}\left(\mathrm{GIC}_{0}\right)(\mathrm{A})$ \\
\hline \multicolumn{5}{|c|}{ Ground model: block 25 node } \\
\hline Pirttikoski & $(66.30 \mathrm{~N}, 27.20 \mathrm{E})$ & 2016 & 285.7 & 81.7 \\
\hline Pikkarala & $(64.90 \mathrm{~N}, 25.80 \mathrm{E})$ & 1291 & 231.6 & 107.9 \\
\hline Sellee & $(65.80 \mathrm{~N}, 24.10 \mathrm{E})$ & 1217 & 184.1 & 56.6 \\
\hline Alajärvi & $(63.00 \mathrm{~N}, 24.20 \mathrm{E})$ & 429 & 186.0 & 110.4 \\
\hline Isoniemi & $(67.50 \mathrm{~N}, 24.80 \mathrm{E})$ & 334 & 188.9 & 41.7 \\
\hline Vyborg & $(60.70 \mathrm{~N}, 28.70 \mathrm{E})$ & 271 & 194.7 & 84.6 \\
\hline Seinäjoki & $(62.80 \mathrm{~N}, 22.70 \mathrm{E})$ & 139 & 108.4 & 74.1 \\
\hline \multicolumn{5}{|c|}{ Ground model: all blocks node } \\
\hline Pirttikoski & $(66.30 \mathrm{~N}, 27.20 \mathrm{E})$ & 2994 & 366.0 & 81.7 \\
\hline Varangerbotn & $(70.00 \mathrm{~N}, 28.50 \mathrm{E})$ & 363 & 302.5 & 35.2 \\
\hline Alapitkä & $(63.20 \mathrm{~N}, 27.50 \mathrm{E})$ & 257 & 237.6 & 84.3 \\
\hline Vuolijoki & $(64.00 \mathrm{~N}, 27.00 \mathrm{E})$ & 51 & 192.9 & 67.0 \\
\hline Utsjoki & $(69.50 \mathrm{~N}, 27.00 \mathrm{E})$ & 50 & 171.0 & 24.8 \\
\hline Seitenoikea & $(64.60 \mathrm{~N}, 28.40 \mathrm{E})$ & 48 & 251.3 & 36.1 \\
\hline
\end{tabular}

of the electric field vectors. Theoretically, GIC could be zero in any power grid if the electric field is everywhere perpendicular to the transmission lines. In practice with realistic grids and electric fields, this never happens as this study demonstrates with a diverse set of electric field configurations.

A consequence and challenge for ideal GIC forecasting are thus to produce not only the correct (regional) magnitude of the electric field, but also to provide a more detailed vector pattern. Although the mean magnitude of the field gives a useful GIC proxy for the grid as a whole, there still remains a large uncertainty of GIC at single substations. On the other hand, this uncertainty can be quantified for a given power grid by performing similar modelling as in this paper. This is always a case-specific task, since power grids and the ground conductivity vary much in different parts of the world. It is also evident that determining the "worst case" spatial pattern of the electric field depends strongly on the power grid in question. 
Concerning vulnerability of a power grid, we cannot make conclusions based only on the magnitudes of GIC at single substations. The vulnerability depends on the type of the transformers whether they tolerate large GIC or not, and on many other technical matters. Furthermore, to analyse effects on a grid as a whole, all substations must be considered. Such a work would require application of dedicated power grid analysis tools, and is beyond the goals of the present study. However, a large set of simulated GIC values such as in our paper would surely provide a valuable input to engineering analyses.

Acknowledgments. We thank all institutes maintaining the IMAGE magnetometer network in North Europe (http://space. fmi.fi/image/). We acknowledge Fingrid Oyj for the long-term collaboration in studies of geomagnetically induced currents in the Finnish high-voltage power grid, and Dr. Antti Pulkkinen for his comments on the manuscript. Comments by the referees and the Editor helped much to clarify the presentation. The editor thanks Tim Divett and an anonymous referee for their assistance in evaluating this paper.

\section{References}

Ádám A, Prácser E, Wesztergom V. 2012. Estimation of the electric resistivity distribution (EURHOM) in the European lithosphere in the frame of the EURISGIC WP2 project. Acta Geod Geophys Hung 47: 377-387. DOI:10.1556/AGeod.47.2012.4.1.

Arajärvi E, Pirjola RJ, Viljanen A. 2011. Effects of neutral point reactors and series capacitors on geomagnetically induced currents in a high-voltage electric power transmission system. Space Weather 9: S11005. DOI:10.1029/2011SW000715.

Bedrosian PA, Love JJ. 2015. Mapping geoelectric fields during magnetic storms: synthetic analysis of empirical United States impedances. Geophys Res Lett 42(10): 160-170. DOI:10.1002/ 2015GL066636.

Beggan CD. 2015. Sensitivity of geomagnetically induced currents to varying auroral electrojet and conductivity models. Earth Planets Space 67: 24. DOI:10.1186/s40623-014-0168-9.

Beggan CD, Beamish D, Richards A, Kelly GS, Thomson AWP. 2013. Prediction of extreme geomagnetically induced currents in the UK high-voltage network. Space Weather 11: 1-13. DOI:10.1002/swe.20065.

Bolduc L. 2002. GIC observations and studies in the Hydro-Québec power system. J Atmos Sol-Terr Phys 64: 1793-1802. DOI:10.1016/S1364-6826(02)00128-1.

Boteler DH, Pirjola RJ, Nevanlinna H. 1998. The effects of geomagnetic disturbances on electrical systems at the earth's surface. Adv Space Res 22: 17-27. DOI:10.1016/S0273-1177(97) 01096-X.

Danskin DW, Lotz SI. 2015. Analysis of geomagnetic hourly ranges. Space Weather 13: 458-468. DOI:10.1002/2015SW001184.

Häkkinen L, Pirjola R, Sucksdorff C. 1989. EISCAT magnetometer cross and theoretical studies connected with the electrojet current system. Geophysica 25(1\&2): 123-134.

Hermance JF, Peltier WR. 1970. Magnetotelluric fields of a line current. J Geophys Res 75: 3351-3356. DOI:10.1029/ JB075i017p03351.

Hibbs RD, Jones FW. 1978. The calculation of perturbation and induction arrows for a three-dimensional conductivity model and various two dimensional source fields. $J$ Geophys Res 83: 5479-5485. DOI:10.1029/JB083iB11p05479.

Langlois P, Bolduc L, Chouteau MC. 1996. Probability of occurrence of geomagnetic storms based on a study of the distribution of the electric field amplitudes measured in Abitibi, Québec, in 1993-94. J Geomag Geoelectr 48: 1033-1041.

Lehtinen M, Pirjola R. 1985. Currents produced in earthed conductor networks by geomagnetically-induced electric fields. Ann Geophys 3: $479-484$.

Love JJ, Rigler EJ, Pulkkinen A, Riley P. 2015. On the lognormality of historical magnetic storm intensity statistics: implications for extreme-event probabilities. Geophys Res Lett 42: 6544-6553. DOI:10.1002/2015GL064842.

Love JJ, Coisson P, Pulkkinen A. 2016. Global statistical maps of extreme-event magnetic observatory $1 \mathrm{~min}$ first differences in horizontal intensity. Geophys Res Lett 43: 4126-4135. DOI:10.1002/2016GL068664.

Molinski TS. 2002. Why utilities respect geomagnetically induced currents. J Atmos Sol-Terr Phys 64: 1765-1778. DOI:10.1016/ S1364-6826(02)00126-8.

Myllys M, Viljanen A, Rui ØA, Ohnstad TM. 2014. Geomagnetically induced currents in Norway: the northernmost high-voltage power grid in the world. J Space Weather Space Clim 4: A10. DOI:10.1051/swsc/2014007.

Ngwira CM, Pulkkinen A, Leila Mays M, Kuznetsova MM, Galvin AB, Simunac K, Baker DN, Li X, Zheng Y, Glocer A. 2013. Simulation of the 23 July 2012 extreme space weather event: what if this extremely rare CME was Earth directed? Space Weather 11: 671-679. DOI:10.1002/2013SW000990.

Ngwira CM, Pulkkinen A, Kuznetsova MM, Glocer A. 2014. Modeling extreme "Carrington-type" space weather events using three-dimensional global MHD simulations. J Geophys Res Space Phys 119: 4456-4474. DOI:10.1002/2013JA019661.

Ngwira CM, Pulkkinen AA, Bernabeu E, Eichner J, Viljanen A, Crowley G. 2015. Characteristics of extreme geoelectric fields and their possible causes: localized peak enhancements. Geophys Res Lett 42: 6916-6921. DOI:10.1002/2015GL065061.

Nikitina L, Trichtchenko L, Boteler DH. 2016. Assessment of extreme values in geomagnetic and geo-electric field variations for Canada. Space Weather 14: 481-494. DOI:10.1002/ 2016SW001386.

Pirjola R. 2010. On the flow of geomagnetically induced currents in an electric power transmission network. Can J Phys 88: 357-363. DOI:10.1139/P10-028.

Pirjola RJ, Häkkinen LVT. 1991. Electromagnetic field caused by an auroral electrojet current system model. In: Kikuchi $\mathrm{H}$, ed. Proceedings of the International URSI Symposium on Environmental and Space Electromagnetics, Tokyo, Japan, September 4-6, 1989. Tokyo: Springer-Verlag, pp. 288-298, Printed in Hong Kong, Chapter 6.5.

Pirjola RJ, Viljanen AT. 1991. Geomagnetic induction in the Finnish $400 \mathrm{kV}$ power system. In: Kikuchi H, ed. Proceedings of the International URSI Symposium on Environmental and Space Electromagnetics, Tokyo, Japan, September 4-6, 1989. Tokyo: Springer-Verlag, pp. 276-287, Printed in Hong Kong, Chapter 6.4.

Pirjola R, Viljanen A. 1989. On geomagnetically-induced currents in the Finnish $400 \mathrm{kV}$ power system by an auroral electrojet current. IEEE Trans Power Deliv 4: 1239-1245. DOI:10.1109/61.25609.

Pirjola R, Boteler D, Viljanen A, Amm O. 2000. Prediction of geomagnetically induced currents in power transmission systems. Adv Space Res 26(1): 5-14. DOI:10.1016/S0273-1177(99)01019-4.

Price AT. 1962. The theory of magnetotelluric methods when the source field is considered. J Geophys Res 67(5): 1907-1918. DOI:10.1029/JZ067i005p01907.

Pulkkinen A, Klimas A, Vassiliadis D, Uritsky V, Tanskanen E. 2006. Spatiotemporal scaling properties of the ground geomagnetic field variations. $J$ Geophys Res 111(A3): A03305. DOI:10.1029/ 2005JA011294. 
Pulkkinen A, Bernabeu E, Eichner J, Beggan C, Thomson AWP. 2012. Generation of 100-year geo-magnetically induced current scenarios. Space Weather 10: S04003. DOI:10.1029/2011SW000750.

Pulkkinen A, Bernabeu E, Eichner J, Viljanen A, Ngwira C. 2015. Regional-scale high-latitude extreme geoelectric fields pertaining to geomagnetically induced currents. Earth Planets Space 67: 93. DOI: $10.1186 / \mathrm{s} 40623-015-0255-6$.

Püthe C, Kuvshinov A. 2013. Towards quantitative assessment of the hazard from space weather. Global 3-D modellings of the electric field induced by a realistic geomagnetic storm. Earth Planets Space 65: 1017-1025. DOI:10.5047/eps.2013.03.003.

Riley P. 2012. On the probability of occurrence of extreme space weather events. Space Weather 10: S02012. DOI:10.1029/ 2011SW000734.

Siscoe GL. 1976. On the statistics of the largest geomagnetic storms per solar cycle. J Geophys Res 81(25): 4782-4784. DOI:10.1029/ JA081i025p04782.

Tanskanen EI. 2009. A comprehensive high-throughput analysis of substorms observed by IMAGE magnetometer network: years 1993-2003 examined. J Geophys Res 114: A05204. DOI:10.1029/ 2008JA013682.

Thomson A, Dawson E, Reay S. 2011. Quantifying extreme behaviour in geomagnetic activity. Space Weather 9: S10001. DOI:10.1029/2011SW000696.

Viljanen A, Pirjola R, Häkkinen L. 1993. An attempt to reduce induction source effects at high latitudes. J Geomag Geoelectr 45: 817-831.

Viljanen A, Pirjola R, Amm O. 1999. Magnetotelluric source effect due to $3 \mathrm{D}$ ionospheric current systems using the complex image method for 1D conductivity structures. Earth Planets Space 51: 933-945. DOI:10.1186/BF03351564.

Viljanen A, Nevanlinna H, Pajunpää K, Pulkkinen A. 2001. Time derivative of the horizontal geomagnetic field as an activity indicator. Ann Geophys 19: 1107-1118. DOI:10.5194/angeo19-1107-2001.

Viljanen A, Pirjola R, Wik M, Adam A, Pracser E, Sakharov Ya, Katkalov Yu. 2012. Continental scale modelling of geomagnetically induced currents. J Space Weather Space Clim 2: A17. DOI: $10.1051 /$ swsc/2012017.

Viljanen A, Pirjola R, Pracser E, Katkalov J, Wik M. 2014. Geomagnetically induced currents in Europe. Modelled occurrence in a continent-wide power grid. J Space Weather Space Clim 4: A09. DOI:10.1051/swsc/2014006.

Viljanen A, Wintoft P, Wik M. 2015. Regional estimation of geomagnetically induced currents based on the local magnetic or electric field. J Space Weather Space Clim 5: A24. DOI:10.1051/ swsc/2015022.

Wang L, Lewis AM, Ogawa Y, Jones WV, Costelloe MT. 2016. Modeling geomagnetic induction hazards using a 3-D electrical conductivity model of Australia. Space Weather 14: 1125-1135. DOI:10.1002/2016SW001436.

Weaver JT. 1973. Induction in a layered plane earth by uniform and non-uniform source fields. Phys Earth Planet Inter 7: 266-281. DOI:10.1016/0031-9201(73)90053-8.

Wei LH, Homeier N, Gannon JL. 2013. Surface electric fields for North America during historical geomagnetic storms. Space Weather 11: 451-462. DOI:10.1002/swe.20073.

Wintoft P, Viljanen A, Wik M. 2016. Extreme value analysis of the time derivative of the horizontal magnetic field and computed electric field. Ann Geophys 34: 1125-1135. 485-491. DOI:10.5194/angeo-34-485-2016.

Zheng K, Pirjola RJ, Boteler DH, Liu L-G. 2013. Geoelectric fields due to small-scale and large-scale source currents. IEEE Trans Power Deliv 28: 442-449. DOI:10.1109/TPWRD.2012.2226248.

Cite this article as: Viljanen A., Pirjola R. 2017. Influence of spatial variations of the geoelectric field on geomagnetically induced currents. J. Space Weather Space Clim. 7: A22 